\title{
Non-invasive assessment of liver fibrosis through FibroMeter in patients with chronic viral hepatitis $B$ and $C$
}

\author{
Oana Săndulescu ${ }^{1,2^{*}}$, Anca Streinu-Cercel ${ }^{1,2}$, Gabriela Ceapraga ${ }^{2}$, Mara Constantinescu' ${ }^{1}$, Adrian Streinu-Cercel ${ }^{1,2}$ \\ From The 10th Edition of the Scientific Days of the National Institute for Infectious Diseases "Prof Dr Matei \\ Bals" \\ Bucharest, Romania. 15-17 October 2014
}

\section{Background}

Since the advent of non-invasive methods for liver fibrosis assessment, liver biopsy has been increasingly replaced with liver stiffness measurements or with computed scores based on serum biomarkers, particularly for monitoring viral infections such as HBV [1] or HCV $[2,3]$.

\section{Methods}

We performed a screening study using FibroMeter (Echosens, Paris) to determine the stage of fibrosis and the necroinflammatory status in a cohort of patients with chronic HBV and HCV infection under surveillance in a tertiary care hospital in Bucharest, Romania.

\section{Results}

We analyzed data from 87 patients, 68 (78.2\%) of which had chronic HBV infection and 19 (21.8\%), chronic HCV infection. The median age was $44.9 \pm 15.0$ (range 17-75). The mean body mass index (BMI) was $26.0 \pm 3.6$ in the HBV group and $26.7 \pm 3.2$ in the HCV group. Overall, 36 patients (41.4\%) had normal BMI, another 36 (41.4\%) had a BMI equivalent for overweight status, and 15 (17.2\%) had grade I obesity.

The distribution of FibroMeter results was: F0-F1: 8 (9.2\%), F1: 2 (2.3\%), F1-F2: 43 (49.4\%), F2: 11 (12.6\%), F2F3: 8 (9.2\%), F3: 7 (8.0\%), F3-F4: 8 (9.2\%). The distribution of the necroinflammatory activity was: A0-A1: 16 (18.4\%), A1-A2: 52 (59.8\%), A2-A3: 19 (21.8\%). We identified no statistically significant differences between patients with HBV and HCV regarding mean fibrosis scores $(\mathrm{p}=0.476)$ or mean necroinflammatory activity scores $(\mathrm{p}=0.681)$.

\section{Conclusion}

The patients included in this study had varied ages and characteristics. FibroMeter classified most of them as F1-F2 but descriptive data should be interpreted in clinical context and potential confounding factors should be identified on a case-by-case basis.

\section{Acknowledgement}

This paper is partially supported by the Sectoral Operational Programme Human Resources Development (SOP HRD), financed from the European Social Fund and by the Romanian Government under the contract numbers POSDRU/159/1.5/S/137390.

\section{Authors' details}

${ }^{1}$ Carol Davila University of Medicine and Pharmacy, Bucharest, Romania. ${ }^{2}$ National Institute for Infectious Diseases "Prof. Dr. Matei Balş", Bucharest, Romania.

Published: 15 October 2014

\section{References}

1. Streinu-Cercel O, Streinu-Cercel A, Preoțescu LL, Streinu-Cercel A: Entecavir as specific antiviral therapy in selected cases of severe acute hepatitis B. GERMS 2012, 2:18-22.

2. Streinu-Cercel A: Hepatitis $C$ in the interferon-free era. GERMS 2013, 3:114.

3. Poynard T, Munteanu M, Deckmyn O, Ngo Y, Drane F, Castille JM, Housset C, Ratziu V, Imbert-Bismut F: Validation of liver fibrosis biomarker (FibroTest) for assessing liver fibrosis progression: proof of concept and first application in a large population. J Hepatol 2012, 57:541-8.

\section{doi:10.1186/1471-2334-14-S7-P50}

Cite this article as: Săndulescu et al:: Non-invasive assessment of liver fibrosis through FibroMeter in patients with chronic viral hepatitis B and C. BMC Infectious Diseases 2014 14(Suppl 7):P50.

\footnotetext{
* Correspondence: oanasandulescu1@gmail.com

${ }^{1}$ Carol Davila University of Medicine and Pharmacy, Bucharest, Romania

Full list of author information is available at the end of the article
} 